\title{
Statistical Monitoring of a Wastewater Treatment Plant: A Case Study
}

\author{
Fouzi Harrou ${ }^{\mathrm{a}}$, Abdelkader Dairi ${ }^{\mathrm{b}}$, Ying Sun $^{\mathrm{a}}$, Mohamed Senouci ${ }^{\mathrm{b}}$ \\ ${ }^{a}$ King Abdullah University of Science and Technology (KAUST) \\ Computer, Electrical and Mathematical Sciences and Engineering (CEMSE) Division, Thuwal 23955-6900, Saudi Arabia, \\ E-mail: fouzi.harrou@kaust.edu.sa \\ ${ }^{b}$ Computer Science Department, University of Oran 1 Ahmed Ben Bella, Algeria \\ Street El senia el mnouer bp 31000 Oran, Algeria. E-mail: dairi.aek@gmail.com
}

\begin{abstract}
The efficient operation of wastewater treatment plants (WWTPs) is key to ensuring a sustainable and friendly green environment. Monitoring wastewater processes is helpful not only for evaluating the process operating conditions but also for inspecting product quality. This paper presents a flexible and efficient fault detection approach based on unsupervised deep learning to monitor the operating conditions of WWTPs. Specifically, this approach integrates a deep belief networks (DBN) model and a one-class support vector machine (OCSVM) to separate normal from abnormal features by simultaneously taking advantage of the feature-extraction capability of DBNs and the superior predicting capacity of OCSVM. Here, the DBN model, which is a powerful tool with greedy learning features, accounts for the nonlinear aspects of WWTPs, while OCSVM is used to reliably detect the faults. The developed DBN-OCSVM approach is tested through a practical application on data from a decentralized WWTP in Golden, CO, USA. The results from the DBNOCSVM are compared with two other detectors: DBN-based K-nearest neighbor and K-means algorithms. The results show the capability of the developed strategy to monitor the WWTP, suggesting that it can raise an early alert to the abnormal conditions.
\end{abstract}

Keywords: Wastewater, learning, OCSVM, DBNs, statistical monitoring, anomaly detection.

\section{Introduction}

Wastewater treatment processes, that aim to remove pollutants from wastewater so that it can be safely reused or discharged, are extremely important for community health and environment. Treated wastewater can be recycled and re-distributed as non-potable water for cleaning, agricultural, and industrial purposes, or safely discharged back into the environment without inducing any serious effects (Grant et al., 2012). Discharges from WWTP must meet the discharge permit limits and the national effluent discharge quality standards to protect the environment and public health (Siegrist, 2017). From a practical point of view, and environmentally speaking, it is often more beneficial to recycle and reuse the treated wastewater rather 
than to discharge it (Castellet and Molinos-Senante, 2016). For example, the reuse of treated wastewater is increasingly becoming a necessity in water-stressed countries, such as countries in the Middle East region, as it offers substantial water - resource savings, while simultaneously providing significant financial benefits, as the costs related to the recycling process are much lower than those of desalting seawater (Dolnicar and Schäfer, 2009; Côté et al., 2005).

To achieve the efficient operation of wastewater treatment plants (WWTPs), some key variables involved in the process, such as dissolved oxygen, nitrogen, phosphorus, and $\mathrm{pH}$, need to be accurately monitored and controlled (Boujelben et al., 2017; Kazor et al., 2016). A good understanding of the WWTP dynamics is required for reliable monitoring and control activities. However, the dynamical behavior of WWTPs is usually complex and uncertain due to nonlinearity, variations in the physical properties in terms of the environmental conditions, strong interactions between the process variables involved, and wide variations in the flow rate and concentration of the composition of the influent of WWTPs. These many factors increase the difficulty of monitoring and control tasks.

Increased attention to modeling wastewater processes has led to the development of several models capable of describing the biological processes involved in WWTPs (e.g., ASM1, ASM2, ASM2d and ASM3) (Henze et al., 2000; Mannina et al., 2011; Plattes et al., 2006). However, these models have complex structures and are comprised of relatively large numbers of parameters that must be identified, making them unsuitable for monitoring purposes. For example, the model ASM1 is comprised of 13 nonlinear differential equations, which involve 19 parameters that are hard to estimate (Dochain and Vanrolleghem, 2001). This high level of model complexity represents a heavy computational burden for the simulation and design process (Vanrolleghem et al., 1999).

Keeping a WWTP running correctly and safely, and generating the desired product quality remains a major challenge in environmental sustainability (Skrjanc and Teslic, 2008; Lee et al., 2003; Steyer et al., 1997). Therefore, monitoring in WWTPs has received special attention from researchers and practitioners in the field of safety engineering. Many methods have been developed for improving fault detection in WWTPs. Wang et al. (2017) proposed a statistical approach based on combined principal components analysis (PCA) and multiple regression to model a WWTP. Dias et al. (2007) applied an artificial neural network (ANN) and neural fuzzy models for monitoring and predicting WWTPs. Wilcox et al. (1995) also used ANN model to monitor and control an anaerobic WWTP. Other researchers focused on using time series analysis approaches to model and monitor WWTPs (Huo et al., 2005; Novotny et al., 1991; 
Capodaglio et al., 1992). Other approaches used latent variable (LV) methods, such as PCA and projection latent structures (PLS), for monitoring and predicting parameters of the influent and effluent of WWTPs. Amaral and Ferreira (2005) applied a PLS regression for activated sludge process (ASP) monitoring. PLS methods have been applied to predict the deterioration of sludge sedimentation properties by monitoring the parameters affecting effluent quality and filamentous bulking in ASPs (Mujunen et al., 1998). PCA and its extensions have been widely used for statistical modeling and monitoring of WWTPs (Liu et al., 2014; Huang et al., 2012; Villez et al., 2008; Lee et al., 2004; Rosén and Lennox, 2001; Lee and Vanrolleghem, 2003).

The main objective of this paper is to enhance the operation and performance of WWTPs through the development of an innovative monitoring strategy. This paper presents a flexible and efficient fault-detection approach based on deep learning to monitor WWTPs. A fault detection strategy capable of dealing with the complex nonlinear dynamics of WWTPs has been proposed. This approach integrates a deep belief network (DBN) model and a one-class support vector machine (OCSVM), and simultaneously takes advantage of the powerful feature-extraction capability of DBNs and the superior predicting capacity of OCSVM. The DBN model, which is a powerful tool with greedy learning features, accounts for the nonlinear aspects of WWTPs, while OCSVM reliably detects the faults in a WWTP dataset. The DBN model, which consists of multiple layers of restricted Boltzmann machines (RBMs), is built via unsupervised greedy layer-wise training using the data collected from a WWTP. Here, WWTP monitoring is addressed as an anomaly-detection problem based on the one-class support vector machine (OCSVM) classifier, trained unsupervised on fault-free data obtained from the DBN model. The central role of the OCSVM classifier is to separate fault-free from faulty data by building a hyperplane. We test the proposed DBN-OCSVM method on practical data collected from a pilot-scale membrane bioreactor (MBR) at the Mines Park Water Reclamation Test Site, a decentralized wastewater treatment facility in Golden, CO.

The remainder of this paper is organized as follows. Section 2 gives a brief overview of machine-learning generative models and the OCSVM algorithm. In Section 3, the proposed DBN-OCSVM fault-detection approach is presented. In Section 4, the performances of the proposed methods are illustrated in a real data application, and Section 5 concludes with a discussion.

\section{Preliminary material}

Over the past two decades, several approaches to fault detection based on shallow learning have been investigated, such as training different classifiers by support vector machines (SVM), AdaBoost, and neural 
networks in supervised learning with one or two layers (Jabeen et al., 2017; Raduly et al., 2007; Ribeiro et al., 2013). However, shallow-learning approaches are not suitable for representing dependencies among multiple variables, are inefficient when dealing with high-dimensionality data, and have a limited ability to model complex functions, leading to unsuitable generalized models. To overcome these limitations, deeplearning approaches have been developed. In a deep-learning approach, several layers are stacked to describe complex functions (Hinton, 2012). In other words, the output of each layer represents the input of the next layer. Using this structure, the classification and learning of complex input information can be achieved. Restricted Boltzmann machines and deep belief networks are powerful deep architectures that overcome most of shallow-learning limitations (Hinton and Salakhutdinov, 2006; Hinton, 2007; Bengio, 2009). In this section, a brief overview of these two models is presented.

\subsection{Restricted Boltzmann Machines (RBMs)}

RBMs are stochastic neural networks (Smolensky, 1986) (see Figure 1) that consist of $m$ visible units $(v \in$ $\left.\{0,1\}^{m}\right)$ and $n$ hidden units $\left(h \in\{0,1\}^{n}\right)$. There are no visible-to-visible or hidden-to-hidden connections, although $v$ and $h$ are fully connected (see Figure 1). These models are trained using contrastive divergence learning procedure based on Gibbs sampling (Hinton, 2012). The learning procedure is comprised of many Gibbs sampling steps (propagate: sample hidden given visibles; reconstruct: sample visible given hidden; repeat) and selecting the weights with the minimum reconstruction error (Salakhutdinov and Hinton, 2009; Bengio, 2009; Hinton et al., 2006).

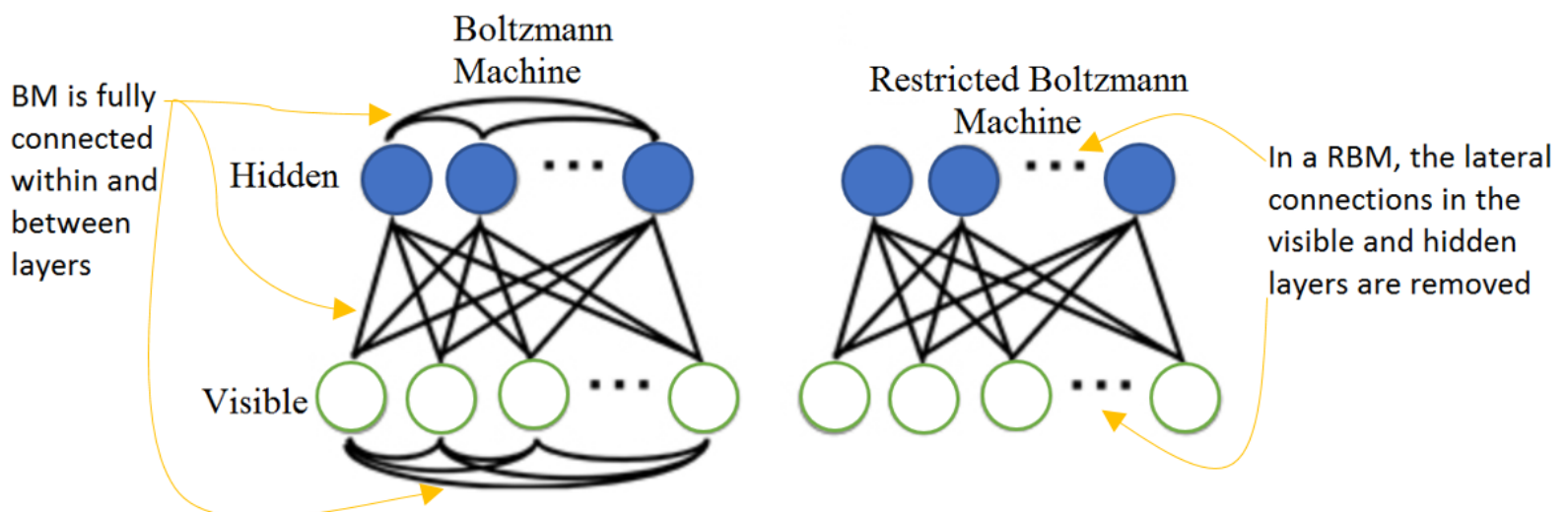

Figure 1: Schematic presentation of a restricted boltzmann machine (RBM). Left: A general Boltzmann machine. Right: An RBM with no visible-to-visible or hidden-to-hidden connections.

RBMs are particularly energy-based models and have been used as generative models for several types of data (Bengio, 2009), such as text, speech, and images. The energy of join configuration is defined 
by (Mohamed et al., 2012):

$$
\operatorname{Energy}(v, h)=-\sum_{i=1}^{m} \sum_{j=1}^{n} W_{i j} v_{i} h_{j},-\sum_{i=1}^{m} b_{i} v_{i}-\sum_{j=1}^{n} c_{j} h_{j}
$$

where $W_{i j}$ is the weight matrix between the visible variable $v_{i}$ and the hidden variable $h_{j}$, and where $b$ and $c$ are the model parameters. The joint distribution of the configuration is given as

$$
\begin{aligned}
P(v, h) & =\frac{1}{\sum_{v} \sum_{h} \exp (-\operatorname{Energy}(v, h))} \exp (-\operatorname{Energy}(v, h)) \\
& =\frac{1}{\sum_{v} \sum_{h} \exp (-\operatorname{Energy}(v, h))} \prod_{i j} e^{W_{i j} v_{i} h_{j}} \prod_{i} e^{b_{i} v_{i}} \prod_{j} e^{a_{j} h_{j}}
\end{aligned}
$$

Since only $v$ is observed, the hidden variables $h$ are marginalized.

$$
P(v)=\sum_{h} \frac{e^{-\operatorname{Energy}(v, h)}}{\sum_{v} \sum_{h} \exp (-\operatorname{Energy}(v, h))}
$$

where $P(v)$ is the probability assigned by the mode to a given visible vector $v$. The hidden nodes are conditionally independent from the visible units, consequently from equation 2 we have:

$$
P(v \mid h)=\prod_{i} p\left(v_{i} \mid h\right), \text { and } P(h \mid v)=\prod_{j} p\left(h_{j} \mid v\right) .
$$

For the binary visible unit $v \in\{0,1\}^{m}$ and the hidden units $h \in\{0,1\}^{n}$, the marginal probability of the RBM is expressed by

$$
\begin{aligned}
& P\left(v_{i}=1 \mid h\right)=\sigma\left(\sum_{j} W_{i j} h_{j}+c_{i}\right), \\
& P\left(h_{j}=1 \mid v\right)=\sigma\left(\sum_{i} W_{i j} v_{i}+b_{j}\right),
\end{aligned}
$$

where $\sigma($.$) is the logistic function and \sigma(x)=(1+\exp (-x))^{-1}$.

The aim of training the RBMs is to adjust the model parameters. This task is achieved by maximizing the probability of the training data under the model. In other words, determination of the parameters (i.e., $w$, $a$ and $b$ ) can be done by maximizing the log-likelihood of the parameters given the training data (Mohamed et al., 2012). 


\subsection{Deep Belief Networks (DBN)}

DBNs are probabilistic generative models that are based on stacked RBMs (see Figure 2). Each layer of DBN is a restricted Boltzmann machine (RBM). The DBN model is first built with unsupervised learning of training data. Then, it is used to predict a new data(O'Connor et al., 2013; Lee et al., 2009; Kang et al., 2013). They are trained in an unsupervised way to extract feature information of the input data. They exhibit high efficiency in discovering layer-by-layer complex nonlinearity. A fast learning procedure for DBN has been developed in Hinton et al. (2006), in which the joint distribution between observed vector $x$ and $\ell$ hidden layers $h^{k}$ is given as (Bengio, 2009),

$$
P\left(x, h^{1}, \ldots, h^{l}\right)=\left(\prod_{k=0}^{\ell-2} P\left(h^{k} \mid h^{k+1}\right)\right) P\left(h^{\ell-1}, h^{\ell}\right)
$$

where $x=h^{0}$ and $P\left(h^{k} \mid h^{k+1}\right)$ is a visible given hidden conditional distribution in an RBM associated with level $k$ of the DBN, and $P\left(h^{\ell-1}, h^{\ell}\right)$ is the joint distribution in the top-level RBM. Indeed, the probability

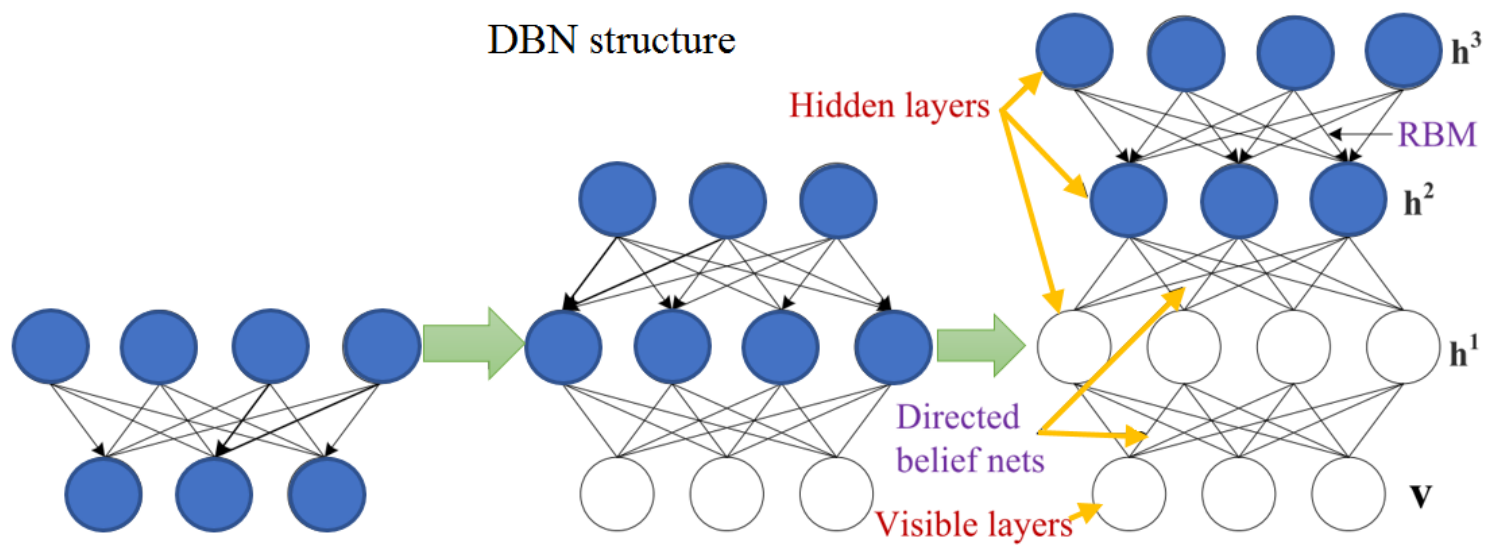

Figure 2: The structure of deep belief networks (DBNs).

of the training data can be increased by stacking more layers to the DBN.In other words, the accuracy of the energy expression is improved by adding more layers to the network (Bengio, 2009).

\subsection{K-nearest neighbor (KNN)}

The k-nearest neighbor (KNN) approach is a non-parametric lazy learning approach, which means that no prior assumptions about the underlying data structure are required (Wu et al., 2008). This property is very useful in situations where the collected data are non-Gaussian distributed or not linearly separable. Classification via a KNN algorithm is performed by evaluating the distances between training samples (e.g., the Euclidean distance function). For a given data point $x$ and a training dataset $\mathcal{D}$, the classification of $x$ is 
achieved based on the KNN that have the smallest distances. The KNN algorithm computes all distances $d_{i}$, defined as $d_{i}=\operatorname{distance}\left(x, \mathcal{D}_{i}\right)$ of the training set $\mathcal{D}$ elements. The KNN algorithm was applied successfully to large datasets, like handwritten digits or satellite image scenes, which proves its capacity to deal with high-dimensionality problems.

\subsection{K-means clustering}

K-means clustering is a partitioning clustering techniquethat minimizes the average squared distance between points in the same cluster (Arthur and Vassilvitskii, 2007) by iteratively assigning $n$ observations to one cluster of $k$ clusters prefixed a priori by centroids. For the desired $k$ clusters, each cluster has its own centroid and data points are assigned to the closest centroid. Following these new assignments, each cluster updates its centroid. This step is repeated until the centroids remain unchanged. Given an integer $k$ and a set of $m$ data points in $Z \subset R^{d}$, the goal is to choose $k$ centers $C$ so that the total squared distance between each point and its closest center, $\varphi=\sum_{z \in Z}^{I} \min _{c \in C}\|z-c\|^{2}$, is minimized.

\subsection{One-class support vector machines (OCSVM)}

The one-class support vector machine (OCSVM) of Schölkopf et al. (2001) is an efficient, unsupervised learning algorithm that learns decision functions for anomaly detection. The one-class classification uses only the normal training data to create a representational model of this data. New data that do not reasonably fit into the model are considered out-of-class (i.e., a fault). The principle of the support vector machines (SVM) is to transform the feature's space into a higher dimensional space using a kernel function (see Figure 3). This transformation allows the obtained data to be approximately linear. Then, the optimal separating hyper plane can be defined in the transformed space for classifying different samples.

The OCSVM maps the outliers in the input space close to the origin of the high-dimensional feature space.

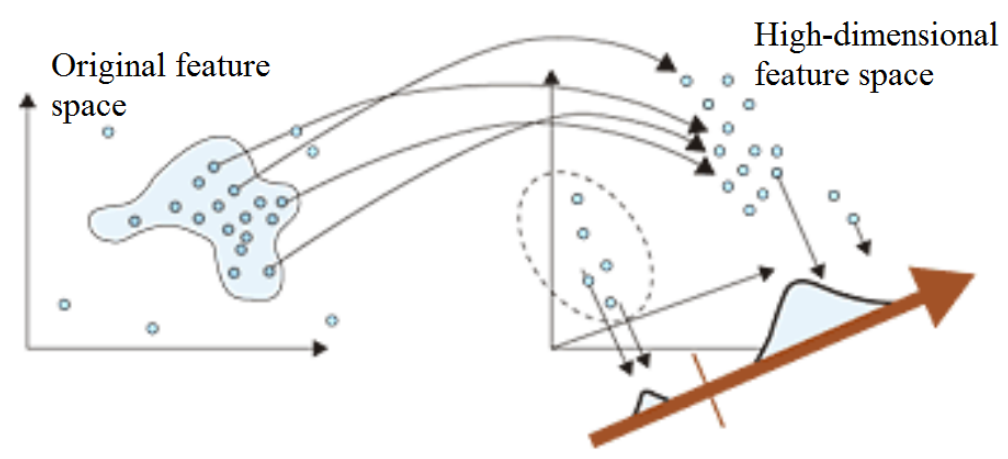

Figure 3: Fault detection via OCSVM algorithm. 
OCSVM is a kernel-based algorithm (see equation 8) where the radial basis function (RBF) (see equation 9) is used to map the input data into a high-dimensional feature space $\mathcal{F}$, the hyperplane that maximizes the margin that best separates the training data from the origin.

$$
\mathcal{K}(x, y)=(\Psi(x) . \Psi(y)),
$$

where $x$ and $y$ are the input vectors, $\Psi$ is a feature map $\mathcal{X} \rightarrow \mathcal{F}$, and $\mathcal{X}$ is set of observed $x$. The RBF kernel is also known as a Gaussian kernel:

$$
\mathcal{K}_{R B F}(x, y)=\exp \left(-\frac{\|x-y\|^{2}}{2 \sigma^{2}}\right) .
$$

The optimal hyperplane for separating the training dataset from the origin is determined by solving the following quadratic optimization problem:

$$
\begin{gathered}
\min _{w \in \mathcal{F}, \xi \in R^{l}, \rho \in R} \frac{1}{2}\|w\|^{2} \frac{1}{\nu l} \sum_{i}^{l} \xi_{i}-\rho, \\
\text { subject to }(w . \Psi(x)) \geq \rho-\xi_{i}, \xi_{i} \geq 0
\end{gathered}
$$

where $\nu \in[0,1]$ is a parameter that characterizes the solution, $w$ is a weight vector, and $\rho$ is an offset. The decision function $f(x)$ can be estimated using equation 11 since the nonzero slack variables $\xi_{i}$ are penalized in the objective function:

$$
f(x)=\operatorname{sgn}((w . \Psi(x))-\rho) .
$$

An hyperplane is constructed based on two parameters $w$ and $\rho$, the distance of all the data points in $\mathcal{F}$ from the hyperplane to the origin.

\section{Combining the DBN model with the OCSVM algorithm (DBN-OCSVM)}

In this section, we present a hybrid fault-detection approach for WWTP monitoring. This approach benefits from both the greedy learning features and dimensionality-reduction of DBNs and the accurate fault detection in multivariate data of the unsupervised OCSVM algorithm (see Figure 4). The proposed DBN-OCSVM approach is implemented in two complementary phases. In the first phase, a DBN model with four layers is constructed using fault-free data. In the second phase, an OCSVM is trained using the features learned by the DBN. In both phases, DBN and OCSVM are trained entirely in an unsupervised 
way.

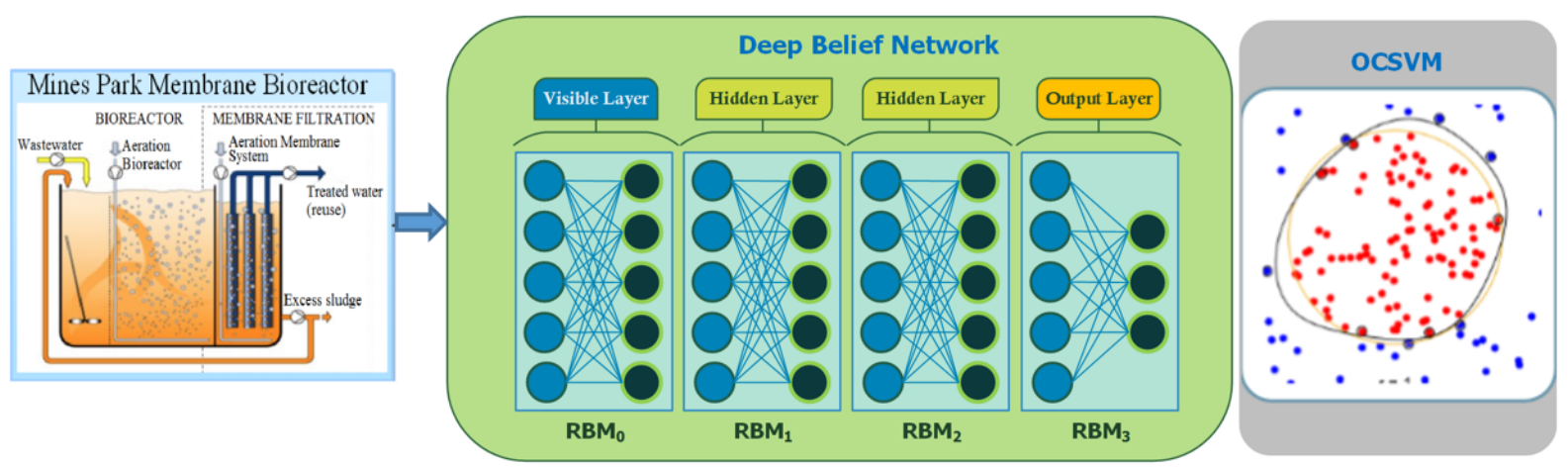

Figure 4: DBN-based OCSVM fault detection approach.

Training of deep belief network: DBNs are highly capable of extracting features from high-dimensional data and of discovering layer-by-layer complex nonlinearity (Bengio et al., 2007). Each layer of the DBN are trained separately. The first layer is trained using data collected from sensors. More hidden units is added to the first layer to increase the flexibility of the DBN model, to discover cross-correlation and data dependencies in the input (visible units), and to learn features. Each layer (hidden) in the DBN uses the generated dataset from the previous layer as input to extract feature information and generate new features that can be used for the next layer. At the end of each layer, the model (i) discovers and extracts new features. After each model (ii), a new encoded output is generated that is used as input for the next layer. This greedy layer-wise training algorithm was proposed by Hinton et al. (2006) for building a DBN model. The output of the last layer from the training samples is used as input to train the OCSVM. Then, the DBN model and OCSVM are used together for testing new datasets.

\section{Real data application: Monitoring a decentralized wastewater treatment plant in Golden CO, USA}

We tested the effectiveness of our approach by using real data collected at the Mines park water reclamation test site, a decentralized wastewater treatment plant located in Golden, CO, USA. This facility process wastewater collected from 400 units of the Mines park student housing complex through a sewer diversion (Prieto et al., 2013)(Figure 5). The system is aimed to produce an effluent quality adequate for landscape irrigation applications (Prieto et al., 2013; Siegrist, 2017). The effluent water has to comply with effluent discharge standards as required by the US EPA and local regulatory agencies (USEPA, 2014; Prieto et al., 2013). Decentralized facilities such as this one will become more common in the future as communities 
begin to reuse their wastewater locally. With greater influent variability, quality, and quantity, decentralized systems will require tighter control and faster response to changes or malfunctions.

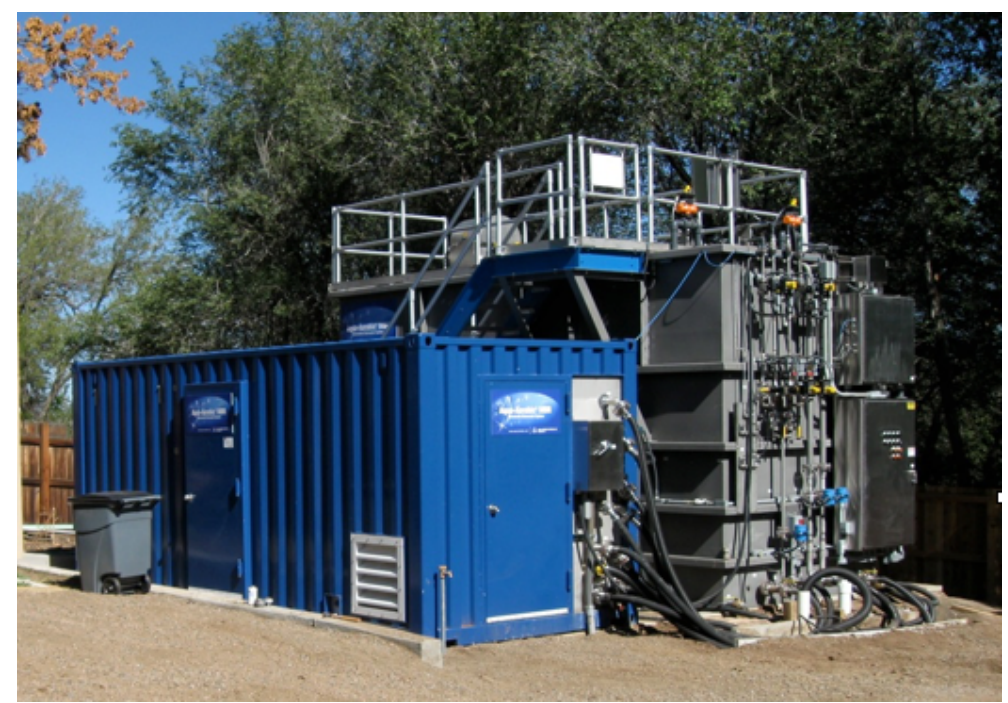

Figure 5: Advanced demo-scale distributed wastewater treatment system at the Mine Park water reclamation research facility, CSM, Golden, Colorado.

This dataset includes 28 variables for which ten-minute averages were recorded from April 10 to May 10, 2010, resulting in a total of 4,464 observations. During this 31-day period, a fault is known to have occurred that affected both $\mathrm{pH}$ and salinity, and the microoganisms in the system took over two months to recover. Here, we select seven key variables involved in the process to monitor: membrane bioreactor (MBR) permeate pressure, MBR dissolved oxygen, permeate turbidity, return activated sludge (RAS) dissolved oxygen content, RAS pH, RAS total suspended solids, and permeate tank conductivity (see Figure 6). The selection of process parameters was made based on experts recommendations using their prior knowledge and experience of this process. One reason that these seven process variables are chosen is that they are spread across the system. The "RAS variables" (i.e., RAS dissolved oxygen (DO), RAS pH and RAS Total Suspended Solids (TSS)) are all related to the water being recirculated through the first set of tanks. The amounts of DO and TSS say a lot about the operation and health of the biological organisms who are responsible for turning the ammonia into nitrogen. The $\mathrm{pH}$ is important because if the $\mathrm{pH}$ drops below a certain level, it will kill all of the biological organisms. The "permeate variables" (i.e., MBR Permeate pressure, Permeate turbidity and Permeate tank conductivity) are all related to the second half of the process, where the water is "permeating" across a membrane. The transmembrane pressure is very important to assess the operation of the membrane, and the turbidity and conductivity are important for assessing how clean the water is coming out of the bioreactors. The concentration of MBR dissolved oxygen 
in the bioreactor tank is a key process parameter that has an important impact on the treatment efficacy and operational cost Siegrist (2017). If DO is too high, it is wasting the electrical energy of the blower; if it is too low, the process may fail. Therefore it's usually monitored as a regularly controlled parameter. The ranges of the process variables to be monitored are given in Table 1.

Table 1: List of monitored variables and their ranges.

\begin{tabular}{l|l|l}
\hline Variable & Range & Unit \\
\hline MBR permeate pressure & 0 to 19.4 & $\mathrm{bar}$ \\
BIO dissolved oxygen & 0 to 4.8 & $\mathrm{mg} / \mathrm{L}$ \\
Permeate turbidity & 0 to 2.9 & $\mathrm{NTU}$ \\
Permeate tank conductivity & 0 to 1000 & $\mu S / \mathrm{m}$ \\
Return activated sludge dissolved oxygen content & 0 to 4.8 & $\mathrm{mg} / \mathrm{L}$ \\
Return activated sludge pH & 0 to 6.8 & - \\
Total suspended solids in return activated sludge & 0 to 6713 & $\mathrm{mg} / \mathrm{L}$ \\
\hline
\end{tabular}

There are two MBRs in this particular system and, since they operate similarly, we only monitor the second one here. The RAS contains active biological organisms that do much of the work of eliminating waste from the water. The design of the system is described in detail in Vuono et al. (2013). The first 500 fault-free observations (shown in Figure 6) are used as training data.

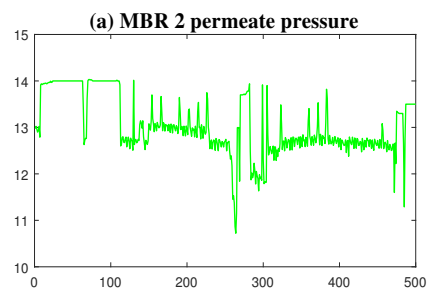

(d) Return activated sludge dissolved

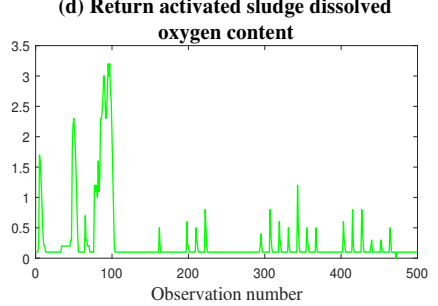

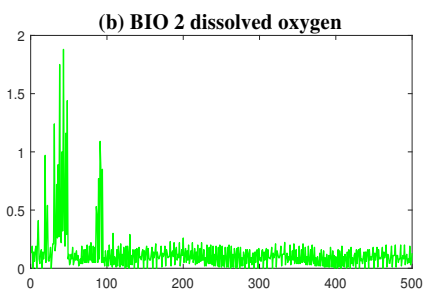
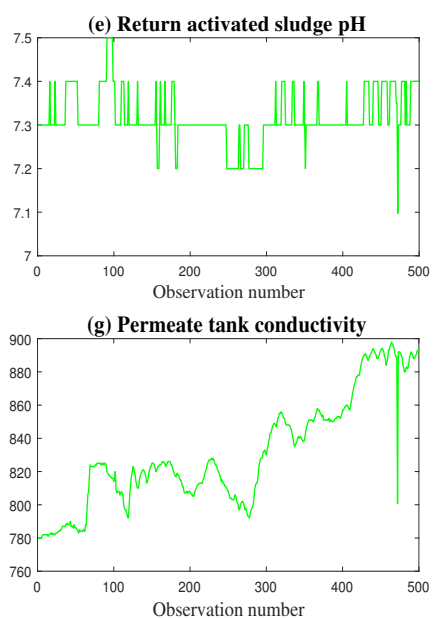

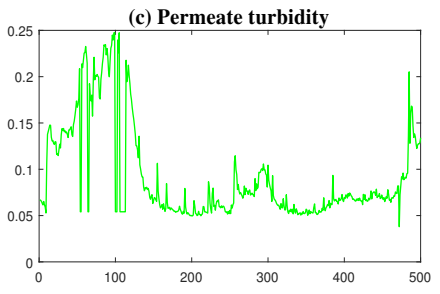

(f) Total suspended solids in return

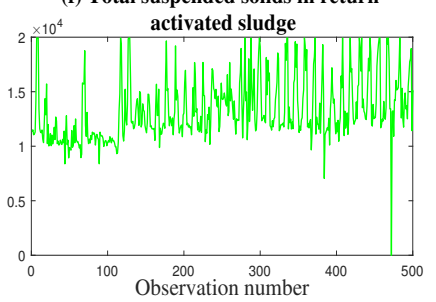

Figure 6: Training data time series of: (a) MBR permeate pressure, (b) BIO 2 dissolved oxygen, (c) permeate turbidity, (d) RAS dissolved oxygen content, (e) RAS pH, (f) RAS total suspended solids, and (g) permeate tank conductivity.

After the DBN model is built using fault-free data, the output features from DBN are used by the OCSVM 
to monitor the MBR system for abnormal events (faults) that may lead the system to depart from its normal state. Indeed, the extracted features from the DBN model are used as the input of the OCSVM algorithm. The output of the last hidden layer of the DBN model is the input of the OCSVM. Specically, the oneclass classier builds boundaries to separate normal (fault-free data) and abnormal (presence of faults) cases. Here, the effectiveness of the proposed DBN-OCSVM fault-detection approach is assessed and compared with DBN-based KNN and K-Means algorithms. The experimental parameters of the machine-learning approaches studied in this paper are presented in Table 2.

Table 2: Parameter settings of the studied approaches.

\begin{tabular}{l|ll}
\hline Models & Parameter & Value \\
\hline \multirow{3}{*}{ DBN } & Layers & 3 \\
& Units & 24 \\
& Learning rate & 0.004 \\
& Epochs & 500 \\
\hline OCSVM & Kernel & RBF \\
RBF & $\gamma$ & 0.1 \\
RBF & $\nu$ & 0.1 \\
\hline K-NN & Leaf size & 30 \\
& Metric & Minkowski \\
& Number of neighbors & 1 \\
& P & 2 \\
& Radius & 1 \\
& Algorithm & BallTree , KDTree \\
\hline K-Means & Number of clusters & 2 \\
& Iteration & 300 \\
& Algorithm & Elkans algorithm (Elkan, 2003) \\
& Initial cluster centers & k-means++ initialization algorithm is used for cluster selection \\
\hline
\end{tabular}

The results from the proposed approaches are shown in Figure 7. To set up a decision threshold for the DBN-KNN approach, we applied an exponentially-weighted moving average (EWMA) monitoring chart to the distances obtained from the KNN algorithm. We used the EWMA chart because of its sensitivity to small changes Zeroual et al. (2017). Figure 7 shows that the DBN-OCSVM, DBN-OCSVM-EWMA, and K-means charts are all able to detect significant anomalies. The proportion of detections is larger when using the DBN-OCSVM and DBN-OCSVM-EWMA charts than with the DBN-K-means. From Figure 7, it can be seen that the three approaches signal alarms starting simultaneously on 18 th April. This can be an evidence that an atypical event worth studying is taking place. This confirms findings from Kazor et al. (2016) where the authors monitored the process using all 28 variables. They applied linear and nonlinear dimension reduction methods (static, dynamic, adaptive, and adaptivedynamic versions of PCA, KPCA, and locally linear embedding (LLE)) to the variables first before constructing $T^{2}$ chart and monitoring it based on nonparametric thresholds. With their method, an alarm would have been issued at 1:40 p.m. 
on April 21st and then again on April 22nd. Here, our method begins to flag the fault even earlier, on April 18th. The initial discovery of the problem by operators on April 24th. Had this abnormal event been detected in time (which is five days prior to when it was actually detected), important degradation of the system could have been avoided, including the two months spent to repair the system. Of course, the choice of OCSVM is motivated by its high performance, in generalization in which the OCSVM can be adjusted to perform as linear or nonlinear algorithm by using nonlinear kernels. As expected, the combination of the greedy learning features of the DBN model whith the OCSVM algorithm showed good performance in detecting faults in the WWTP application.
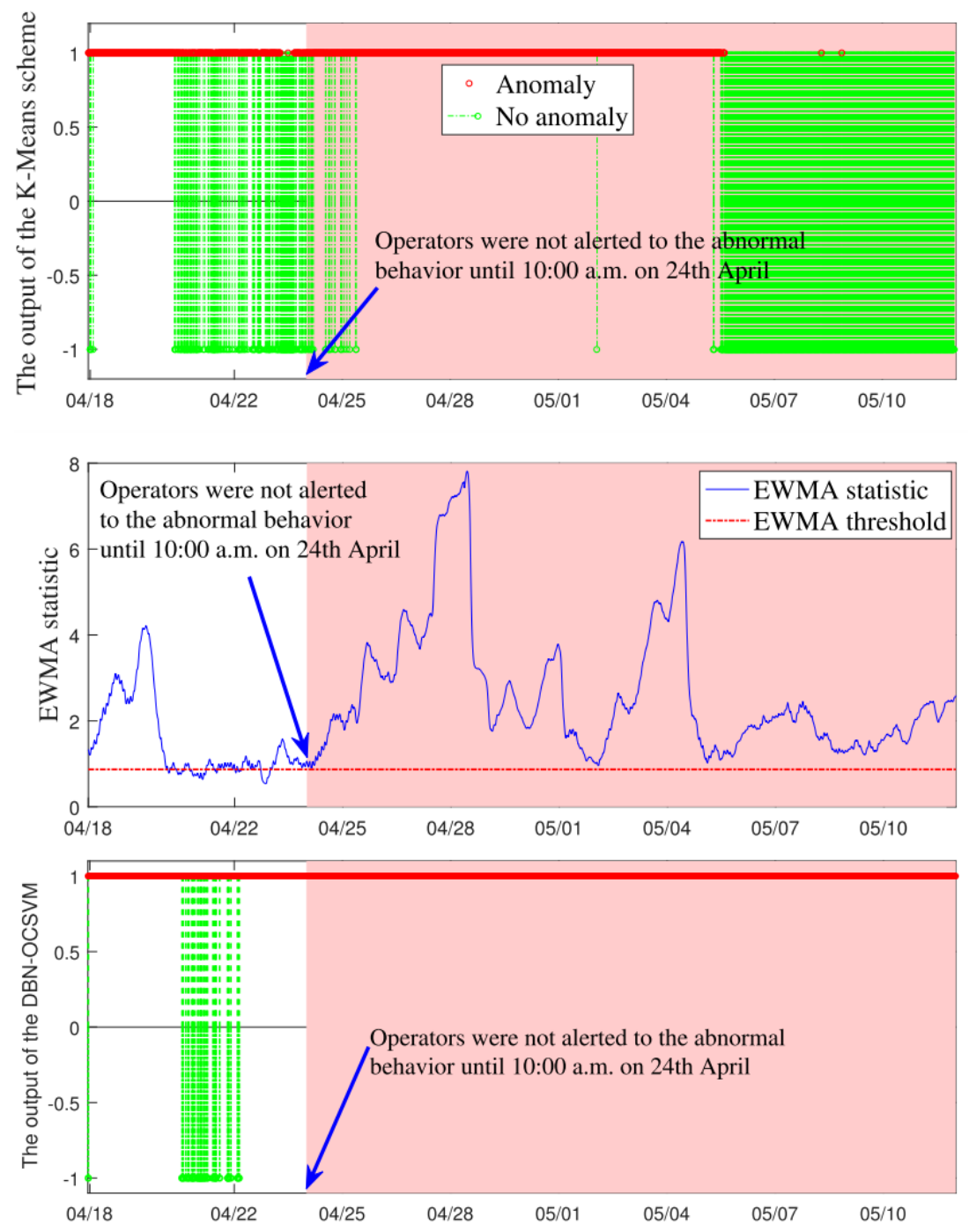

Figure 7: Monitoring results from the DBN-K-means approach (top), the DBN-KNN-EWMA approach (middle), and the DBN-OCSVM (bottom) for $\mathrm{pH}$ data set. 


\section{Conclusion}

This paper proposes a development of an unsupervised machine-learning method for detecting potential faults in wastewater treatment plants (WWTPs). Specifically, the developed approach uses deep belief networks (DBNs) to model the complex features of data from WWTPs and employs a one-class support vector machines (OCSVM) to separate normal from abnormal features. We evaluated the proposed DBNOCSVM approach using practical data from a decentralized WWTP in Golden, CO, USA. Also, we compared the detection quality of our DBN-OCSVM approach to that of K-NN-EWMA and K-Means algorithms and found better performance.

In the presence of highly noisy measurements, the quality of any detection algorithm will be affected. When the data is very noisy, false alarms might be generated during the fault detection task. Therefore, it is crucial to develop a robust approach that can handle noisy data. In fact, wavelet-based multiscale representation of data has been demonstrated to provide effective noise-feature separation in the data and to approximately decorrelate auto-correlated data. As future work, we plan to develop a statistical approach that can handle noisy data by exploiting the advantages of multiscale representation of data and those of the deep learning-based fault detection approaches to better detect faults.

\section{Acknowledgement}

This publication is based upon work supported by the King Abdullah University of Science and Technology (KAUST) Office of Sponsored Research (OSR) under Award No: OSR-2015-CRG4-2582. We are grateful to the three referees, the Associate Editor, and the Editor-in-Chief for their comments.

\section{References}

Amaral, A., Ferreira, E., 2005. Activated sludge monitoring of a wastewater treatment plant using image analysis and partial least squares regression. Analytica Chimica Acta 544 (1), 246-253.

Arthur, D., Vassilvitskii, S., 2007. k-means++: The advantages of careful seeding. In: Proceedings of the eighteenth annual ACM-SIAM symposium on Discrete algorithms. Society for Industrial and Applied Mathematics, pp. 1027-1035.

Bengio, Y., 2009. Learning deep architectures for AI. Foundations and trends® in Machine Learning 2 (1), 1-127.

Bengio, Y., Lamblin, P., Popovici, D., Larochelle, H., 2007. Greedy layer-wise training of deep networks. In: Advances in neural information processing systems. pp. 153-160.

Boujelben, I., Samet, Y., Messaoud, M., Makhlouf, M. B., Maalej, S., 2017. Descriptive and multivariate analyses of four tunisian wastewater treatment plants: A comparison between different treatment processes and their efficiency improvement. Journal of environmental management $187,63-70$ 
Capodaglio, A., Novotny, V., Fortina, L., 1992. Modelling wastewater treatment plants through time series analysis. Environmetrics 3 (1), 99-120.

Castellet, L., Molinos-Senante, M., 2016. Efficiency assessment of wastewater treatment plants: A data envelopment analysis approach integrating technical, economic, and environmental issues. Journal of environmental management 167, $160-166$.

Côté, P., Siverns, S., Monti, S., 2005. Comparison of membrane-based solutions for water reclamation and desalination. Desalination $182(1-3), 251-257$.

Dias, A., Alves, M., Ferreira, E., 2007. Application of computational intelligence techniques for monitoring and prediction of biological wastewater treatment systems. In: In proceedings of the Int. IWA Conf. on Automation in Water Quality monitoring, 3:Gent, Belgium. Springer, pp. 1-8.

Dochain, D., Vanrolleghem, P., 2001. Dynamical Modelling and Estimation in Wastewater Treatment Processes. IWA publishing.

Dolnicar, S., Schäfer, A. I., 2009. Desalinated versus recycled water: public perceptions and profiles of the accepters. Journal of Environmental Management 90 (2), 888-900.

Elkan, C., 2003. Using the triangle inequality to accelerate K-Means. In: Proceedings of the 20th International Conference on Machine Learning (ICML-03). pp. 147-153.

Grant, S. B., Saphores, J.-D., Feldman, D. L., Hamilton, A. J., Fletcher, T. D., Cook, P. L., Stewardson, M., Sanders, B. F., Levin, L. A., Ambrose, R. F., 2012. Taking the "waste" out of "wastewater" for human water security and ecosystem sustainability. Science 337 (6095), 681-686.

Henze, M., Gujer, W., Mino, T., Van Loosdrecht, M., 2000. Activated sludge models ASM1, ASM2, ASM2d and ASM3. IWA publishing WA, London.

Hinton, G. E., 2007. Learning multiple layers of representation. Trends in cognitive sciences 11 (10), 428-434.

Hinton, G. E., 2012. A practical guide to training restricted boltzmann machines. In: Neural networks: Tricks of the trade. Springer, pp. 599-619.

Hinton, G. E., Osindero, S., Teh, Y. W., 2006. A fast learning algorithm for deep belief nets. Neural computation 18 (7), 1527-1554.

Hinton, G. E., Salakhutdinov, R. R., 2006. Reducing the dimensionality of data with neural networks. Science 313 (5786), $504-507$.

Huang, M., Ma, Y., Wan, J., Zhang, H., Wang, Y., 2012. Modeling a paper-making wastewater treatment process by means of an adaptive network-based fuzzy inference system and principal component analysis. Industrial \& Engineering Chemistry Research 51 (17), 6166-6174.

Huo, J., Seaver, W., Robinson, R., Cox, C., 2005. Application of time series models to analyze and forecast the influent components of wastewater treatment plants (WWTPs). In: Impacts of Global Climate Change. pp. 1-11.

Jabeen, A., Ahmad, N., Raza, K., 2017. Machine learning-based state-of-the-art methods for the classification of RNA-seq data. bioRxiv, 120592 .

Kang, S., Qian, X., Meng, H., 2013. Multi-distribution deep belief network for speech synthesis. In: Acoustics, Speech and Signal Processing (ICASSP), 2013 IEEE International Conference on. IEEE, pp. 8012-8016.

Kazor, K., Holloway, R. W., Cath, T. Y., Hering, A. S., 2016. Comparison of linear and nonlinear dimension reduction techniques for automated process monitoring of a decentralized wastewater treatment facility. Stochastic environmental research and risk assessment $30(5), 1527-1544$. 
Lee, D., Vanrolleghem, P., 2003. Monitoring of a sequencing batch reactor using adaptive multiblock principal component analysis. Biotechnology and Bioengineering 82 (4), 489-497.

Lee, H., Pham, P., Largman, Y., Ng, A. Y., 2009. Unsupervised feature learning for audio classification using convolutional deep belief networks.

URL http://papers.nips.cc/paper/3674-unsupervised-feature-learning-for-audio-classification-usingconvolutional-deep-belief-networks.pdf, Last accessed 24 March 2018.

Lee, J.-M., Yoo, C., Choi, S., Vanrolleghem, P., Lee, I.-B., 2004. Nonlinear process monitoring using kernel principal component analysis. Chemical engineering science 59 (1), 223-234.

Lee, J.-M., Yoo, C.-K., Lee, I.-B., 2003. New monitoring technique with an ICA algorithm in the wastewater treatment process. Water Science and Technology 47 (12), 49-56.

Liu, Y., Pan, Y., Sun, Z., Huang, D., 2014. Statistical monitoring of wastewater treatment plants using variational Bayesian PCA. Industrial \& Engineering Chemistry Research 53 (8), 3272-3282.

Mannina, G., DiTrapani, D., Viviani, G., Ødegaard, H., 2011. Modelling and dynamic simulation of hybrid moving bed biofilm reactors: model concepts and application to a pilot plant. Biochemical engineering journal 56 (1), 23-36.

Mohamed, A.-R., Dahl, G. E., Hinton, G., 2012. Acoustic modeling using deep belief networks. IEEE Transactions on Audio, Speech, and Language Processing 20 (1), 14-22.

Mujunen, S.-P., Minkkinen, P., Teppola, P., Wirkkala, R.-S., 1998. Modeling of activated sludge plants treatment efficiency with PLSR: a process analytical case study. Chemometrics and Intelligent Laboratory Systems 41 (1), 83-94.

Novotny, V., Jones, H., Feng, X., Capodaglio, A., 1991. Time series analysis models of activated sludge plants. Water Science and Technology 23 (4-6), 1107-1116.

O'Connor, P., Neil, D., Liu, S.-C., Delbruck, T., Pfeiffer, M., 2013. Real-time classification and sensor fusion with a spiking deep belief network. Frontiers in neuroscience 7 .

Plattes, M., Henry, E., Schosseler, P., Weidenhaupt, A., 2006. Modelling and dynamic simulation of a moving bed bioreactor for the treatment of municipal wastewater. Biochemical Engineering Journal 32 (2), 61-68.

Prieto, A., Vuono, D., Holloway, R., Benecke, J., Henkel, J., Cath, T., Reid, T., Johnson, L., Drewes, J., 2013. Decentralized wastewater treatment for distributed water reclamation and reuse: The good, the bad, and the uglyexperience from a case study. In: Novel Solutions to Water Pollution. ACS Publications, pp. 251-266.

Raduly, B., Gernaey, K. V., Capodaglio, A. G., Mikkelsen, P. S., Henze, M., 2007. Artificial neural networks for rapid WWTP performance evaluation: Methodology and case study. Environmental Modelling \& Software 22 (8), 1208-1216.

Ribeiro, D., Sanfins, A., Belo, O., 2013. Wastewater treatment plant performance prediction with support vector machines. In: Industrial Conference on Data Mining. Springer, pp. 99-111.

Rosén, C., Lennox, J., 2001. Multivariate and multiscale monitoring of wastewater treatment operation. Water research 35 (14), $3402-3410$.

Salakhutdinov, R., Hinton, G., 2009. Deep Boltzmann machines. In: Artificial Intelligence and Statistics. pp. 448-455.

Schölkopf, B., Platt, J. C., Shawe-Taylor, J., Smola, A. J., Williamson, R. C., 2001. Estimating the support of a high-dimensional distribution. Neural computation 13 (7), 1443-1471.

Siegrist, R. L., 2017. Decentralized Water Reclamation Engineering. Springer.

Skrjanc, I., Teslic, L., 2008. Monitoring of waste-water treatment plant using Takagi-Sugeno fuzzy model. In: The 14th IEEE Mediterranean Electrotechnical Conference, MELECON 2008. IEEE, pp. 67-70. 
Smolensky, P., 1986. Information processing in dynamical systems: Foundations of harmony theory; cu-cs-321-86.

Steyer, J.-P., Rolland, D., Bouvier, J.-C., Moletta, R., 1997. Hybrid fuzzy neural network for diagnosis-application to the anaerobic treatment of wine distillery wastewater in a fluidized bed reactor. Water Science and Technology 36 (6-7), 209217.

USEPA, 2014. EPA regulations (2014) 40 CFR: protection of the environment. Subchapter N-"Effluent guidelines and standards. Parts 400-424 and 425-471".

Vanrolleghem, P., Spanjers, H., Petersen, B., Ginestet, P., Takacs, I., 1999. Estimating (combinations of) activated sludge model No. 1 parameters and components by respirometry. Water Science and Technology 39 (1), $195-214$.

Villez, K., Ruiz, M., Sin, G., Colomer, J., Rosen, C., Vanrolleghem, P., 2008. Combining multiway principal component analysis (MPCA) and clustering for efficient data mining of historical data sets of SBR processes. Water Science and Technology 57 (10), 1659-1666.

Vuono, D., Henkel, J., Benecke, J., Cath, T., Reid, T., Johnson, L., Drewes, J., 2013. Flexible hybrid membrane treatment systems for tailored nutrient management: A new paradigm in urban wastewater treatment. Journal of membrane science $446,34-41$.

Wang, X., Ratnaweera, H., Holm, J., Olsbu, V., 2017. Statistical monitoring and dynamic simulation of a wastewater treatment plant: A combined approach to achieve model predictive control. Journal of Environmental Management 193, 1-7.

Wilcox, S., Hawkes, D., Hawkes, F., Guwy, A., 1995. A neural network, based on bicarbonate monitoring, to control anaerobic digestion. Water Research 29 (6), 1465-1470.

Wu, X., Kumar, V., Quinlan, J. R., Ghosh, J., Yang, Q., Motoda, H., McLachlan, G. J., Ng, A., Liu, B., Philip, S. Y., et al., 2008. Top 10 algorithms in data mining. Knowledge and information systems 14 (1), 1-37.

Zeroual, A., Harrou, F., Sun, Y., Messai, N., 2017. Monitoring road traffic congestion using a macroscopic traffic model and a statistical monitoring scheme. Sustainable Cities and Society 35, 494-510. 